\title{
Analysis of threshed rice mixture separation through vibration screen using discrete element method
}

\author{
Li Hua ${ }^{1}$, Wang Jinshuang ${ }^{2}$, Yuan Jianbo ${ }^{3}$, Yin Wenqing ${ }^{1 *}$, \\ Wang Zhiming ${ }^{2}$, Qian Youzhang ${ }^{1}$ \\ (1. College of Engineering, Nanjing Agricultural University, Nanjing 210095, China; \\ 2. Jinhua Vocational Technical College, Jinhua 321000, Zhejiang, China; 3. Nanjing Tech University, Nanjing 210093, China)
}

\begin{abstract}
The discrete element method was utilized to construct three-dimensional discrete element models for the rice mixture, and their motions were analyzed numerically on a planar vibration screening device. The results showed that, after falling onto the vibrating screen surface, the mixture undergoes a reciprocating motion within the same cycle. During the screening process, the mixture undergoes segregation, slides along the screen surface, passes through the screen and then falls. In comparing the movement of grains and shriveled grains, it can be seen that the velocity of shriveled grains experiences cyclical changes, which is consistent with the grains' motion cycle. The impact on grains is shown to be greater than that on shriveled grains, and the average speeds of shriveled grains and grains converge. The curve shows that the screening of repetitive movements has a significant effect on the average velocity of shriveled grains, but also the velocity of shriveled grains and the vibration parameters can be well represented by a fitting equation. It is beneficial for the separation of grains from shriveled grains to choose a greater vibration frequency and screen surface inclination in the range of commonly used.
\end{abstract}

Keywords: rice mixture separation, vibration screen, discrete element method, numerical computation, mechanism DOI: $10.25165 / \mathrm{j}$.jjabe.20171006.2910

Citation: Li H, Wang J S, Yuan J B, Yin W Q, Wang Z M, Qian Y Z. Analysis of threshed rice mixture separation through vibration screen using discrete element method. Int J Agric \& Biol Eng, 2017; 10(6): 231-239.

\section{Introduction}

China is the world's largest rice producing country, and the rice harvest is highly dependent on the efficiency of the harvester for simultaneously completing the cutting,

Received date: 2016-10-10 Accepted date: 2017-01-26

Biographies: Li Hua, PhD, Professor, research focuses on agricultural mechanization and biomass energy, Email: lihua@njau.edu.cn; Wang Jinshuang, Master, Associate Professor, research focuses on agricultural harvesting equipment, Email: 791228154@qq.com; Yuan Jianbo, Master student, research focuses on modern agricultural machinery equipment, Email: 335943408@qq.com; Wang Zhiming, PhD, Associate Professor, research focuses on modern agricultural machinery equipment, Email: jhcwzm@163.com; Qian Youzhang, Master, research focuses on modern agricultural machinery equipment, Email: 1451663029@qq.com.

*Corresponding author: Yin Wenqing, $\mathrm{PhD}$, Professor, research focuses on agricultural mechanization and automation. College of Engineering, Nanjing Agricultural University, No. 40, Dianjiangtai Road, Pukou Nanjing 210095, Jiangsu, China. Tel: +8613851938631, Fax:+86-25-58606699, Email: 713709@qq.com. threshing, cleaning and separation work during harvest. A plane vibratory screening device is an important component of the modern combine harvester and has a significant influence on the separation performance. The screening process of the threshed mixture (which contains short straws, grains, shriveled grains and crushed leaves) is a multiple stochastic discrete process. Relying on the powerful computing capabilities of modern computers, the microscopic behavior of screening as well as the screening mechanism can be explored based on the discrete element method (DEM). This process has attracted much research attention.

Cleary and Sawley ${ }^{[1,2]}$ used the discrete element method and a three-dimensional (3D) spherical model to conduct a DEM simulation of the particle mixing as well as the screening process on a screen surface in the vertical vibration direction. They analyzed the screening mechanism when particles underwent vibration screening 
and found that DEM can be used as an auxiliary design tool for screening equipment. Furthermore, Li et al. ${ }^{[3,4]}$ applied the two-dimensional circular DEM model and utilized a stationary tilting screen surface to simulate the screening process of the granular assembly of the different binary grain sizes, and examine the movement process and separation mechanism of particle groups on the plane sieve for discussing the impact of various factors on screening efficiency. Using the 3D discrete element method, Chen and Tong ${ }^{[5]}$ simulated the collision of particles on the vibrating surface in screening process and examined the effects of vibration frequency and screen surface length on the screening efficiency. Jiao et al. ${ }^{[6]}$ set up a DEM screening system using $\mathrm{VC}+$ and achieved a dynamic simulation of vibration screening. Wiącek $^{[7]}$ determined influences of the particle shape and friction on the mechanical response of a granular assembly in a uniaxial compression through DEM simulations and laboratory experiments with pea bean seeds. Zhao et al. $^{[8,9]}$ used DEM to conduct a 3D simulation on the vibratory screening process of minerals to explore the ore screening mechanism and study the influence of vibratory screen motion parameters on screening efficiency. Jiao and Zhao ${ }^{[10]}$ used the DEM method to conduct a numerical calculation and simulation on the vibratory screening of rice and brown rice, and compared with the experimental results which demonstrated that the numerical computation was feasible. Lenaerts et al. ${ }^{[11]}$ introduced modified DEM for better fitting simulations of the grain-straw separation, which is one of the most critical processes in combine harvesters.

Among studies involving numerical simulations of grain screening, many scholars overlooked the effect that particle shape can have on screening. In such studies, a spherical particle model is typically adopted, which exists a significant difference in actual situations on the ratio of the mixture's different components and the screen surface size parameters. In fact, minimal research has been conducted on comparing the movement patterns of different components in the screening process, and there is little in-depth study on the movement of the granular assembly and the screening mechanism.

In this paper, the screening process of the grain mixture on the vibratory sieve was reproduced through numerical calculations of different components on the sieve using the soft ball model of 3D discrete element Through simulation experiments, the average impact and the average velocity of grains and shriveled grains during screening was studied to reveal their movement patterns on the screen surface and vibration screening mechanism.

\section{Multi-component discrete element model and simulation parameters}

\subsection{Discrete element contact mechanics model}

The soft ball contact model by modified discrete element method (MDEM) was adopted as shown in Figure 1.

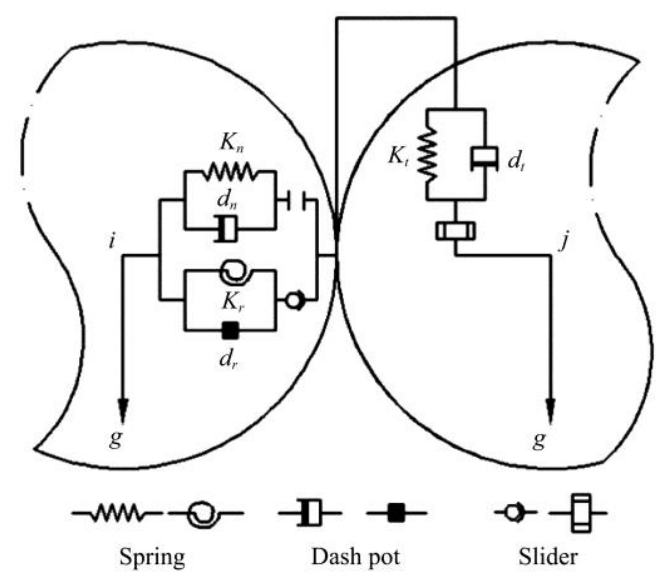

Figure 1 Contact model

In Figure $1, K_{n}, K_{t}$ and $K_{r}$ are normal stiffness, tangential stiffness and roll stiffness, respectively, while $d_{n}, d_{t}$ and $d_{r}$ are the damping for normal stiffness, tangential stiffness and roll stiffness, respectively. Particles are subject to gravity in addition to the normal and tangential contact force between particles. Furthermore, they are also subject to the moment caused by the tangential force as well as the rolling friction moment. Therefore, the motion ${ }^{[12]}$ of the $i^{\text {th }}$ particles can be expressed by the following two equations:

$$
\begin{gathered}
m_{i} \frac{d V_{i}}{d t}=m_{i} g+\sum_{j=1}^{n_{i}}\left(F_{n, i j}+F_{t, i j}\right) \\
I_{i} \frac{d \omega_{i}}{d t}=\sum_{j=1}^{n_{i}}\left(T_{t, i j}+T_{r, i j}\right)
\end{gathered}
$$

where, $m_{i}$ is the mass of the particle $i ; I_{i}$ is the moment of inertia of the particle $i ; n_{i}$ is the total number of particles that have contact with the particle $i ; g$ is the gravitational acceleration; $V_{i}$ is the moving speed of the particle $i ; t$ is 
the time step; and $\omega_{i}$ is the angular velocity of the particle

$i$. The normal force $F_{n, i j}$, the tangential force $F_{t, i j}$, the rolling friction torque $T_{r, i j}$, and the tangential torque $T_{t, i j}$ can be obtained by using the basic Newtonian mechanics formula ${ }^{[8]}$. Each individual particle simultaneously moves and rolls under the action of the above forces and moments.

\subsection{D discrete element model for threshed grain mixture}

In the previous study, the research team constructed a $3 \mathrm{D}$ CAD model of a threshed grain mixture ${ }^{[13]}$. As a result, the bonded particle method ${ }^{[14,15]}$ was used in this study to represent the non-spherical shape of three kinds of extractions with multiple spheres adhered together. The physical properties of the adhered spheres were the same as the corresponding mixture. Relying on this method, a 3D discrete element model of the rice grain mixture was constructed as shown in Figure 2.

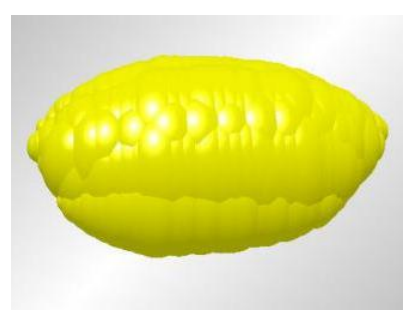

a. Grain

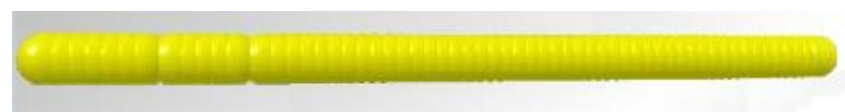

c. A short stalk

Figure 2 3D DEM model of threshed mixture

\subsection{Model of vibrating screen}

Louver sieves are usually applied to the screening systems of small combined harvesters. Because of the sieve's reciprocating motion and wind force, the threshed mixture grains can be separated when they fall onto the screening surface. In order to save computation time, some parts not in contact with the grains were removed. The simplified vibrating screen is shown in Figure 3. Additionally, the fish scale of chaffer opening is $20^{\circ}$, the size of fish scale is $8 \mathrm{~mm} \times 8 \mathrm{~mm}$, the length of the sifter is $1000 \mathrm{~mm}$ and the width of the sifter is $300 \mathrm{~mm}$.

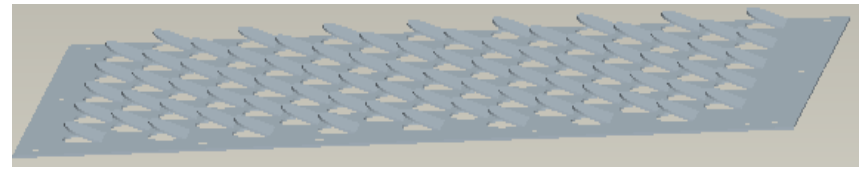

Figure 3 Simplified vibrating screen

\subsection{Simulation software and simulation}

The discrete element analysis tool from EDEM2.3 was used for the simulation. In the simulation, since the sifter is a moving part, it was set to perform a linear back and forth motion ${ }^{[16]}$. In the particle mixture used for the simulation, the ratio of grains, shriveled grains and short stalks produced per second was assumed to be 793:205:2 $2^{[17]}$, and the step length was 0.02 s. The components and physical parameters of the granular material were set as shown in Table 1, and the physical parameters for the interactions between particles are shown in Table $2^{[18,19]}$.

Table 1 Physical parameters of granular materials

\begin{tabular}{cccc}
\hline Material & Density $/ \mathrm{kg} \cdot \mathrm{m}^{-3}$ & Shear modulus $/ \mathrm{Pa}$ & Poisson's ratio \\
\hline Steel & 7850 & $1 \times 10^{10}$ & 0.30 \\
Grain & 1280 & $2.1 \times 10^{5}$ & 0.25 \\
Short stalk & 160 & $4.4 \times 10^{3}$ & 0.45 \\
Shriveled grain & 190 & $2.0 \times 10^{5}$ & 0.35 \\
\hline
\end{tabular}

Table 2 Physical parameters of particle interactions

\begin{tabular}{cccc}
\hline & $\begin{array}{c}\text { Coefficient of } \\
\text { rolling friction }\end{array}$ & $\begin{array}{c}\text { Coefficient of } \\
\text { static friction }\end{array}$ & $\begin{array}{c}\text { Coefficient of } \\
\text { restitution }\end{array}$ \\
\hline Grain-grain & 0.25 & 0.75 & 0.43 \\
Grain-stalk & 0.17 & 0.50 & 0.36 \\
Grain-shriveled grain & 0.28 & 0.84 & 0.42 \\
Grain-steel & 0.12 & 0.35 & 0.45 \\
Shriveled-shriveled grain & 0.31 & 0.93 & 0.38 \\
Shriveled-steel & 0.18 & 0.53 & 0.39 \\
Shriveled-stalk & 0.24 & 0.77 & 0.31 \\
Stalk-stalk & 0.15 & 0.46 & 0.22 \\
Stalk-steel & 0.10 & 0.26 & 0.29 \\
\hline
\end{tabular}

\subsection{Experimental design}

In order to study the screening mechanism of the granular assembly under different vibration parameters, an experiment focused on numerical simulations under examining single parameter based upon the sieve's motion parameters during vibratory screening. As shown in Table 3, four levels were set for each of the parameters, and numerical calculations were made using various combinations.

Table 3 Grouped experiments examining single parameters

\begin{tabular}{cccc}
\hline Experiment No. & Factor & Level & Conditions \\
\hline $1-4$ & Amplitude/mm & $20,25,30,35$ & $\alpha=4^{\circ}, f=4 \mathrm{~Hz}$ \\
$5-8$ & Frequency $/ \mathrm{Hz}$ & $3,4,5,6$ & $\alpha=4^{\circ}, A=25 \mathrm{~mm}$ \\
$8-12$ & $\begin{array}{c}\text { Sieve surface } \\
\text { inclination } /\left(^{\circ}\right)\end{array}$ & $3,4,5,6$ & $A=25 \mathrm{~mm}, f=4 \mathrm{~Hz}$ \\
\hline
\end{tabular}




\section{Experiment verification}

\subsection{Cleaning testing platform}

The experimental platform is shown in Figure 4. The vibration sieve was driven by the electromotor and its speed was controlled by the converter. When the threshed mixture grain fell onto the screening room, the mixture grain could be separated by the combined action of the wind force and the vibration force. The speed of the fan could also be changed by another converter. In order to analyze the distribution of undersized grain, the flow board was set up, and the undersized grain could therefore fall into five sample boxes.

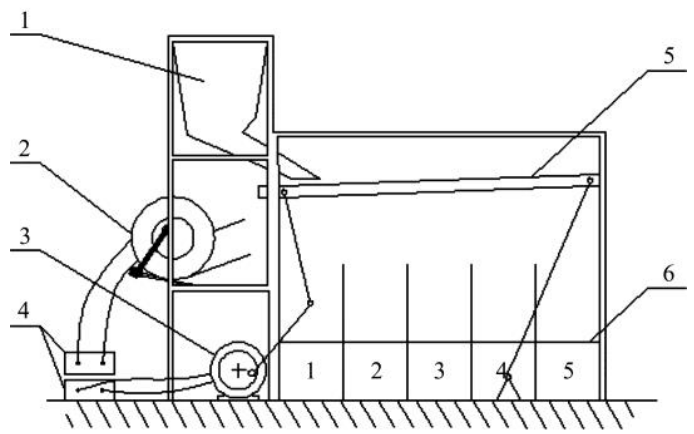

1. Hopper 2. Fan 3. Electro motor 4. Converter 5. Vibration sieve 6. Sample box

Figure 4 Cleaning device

\subsection{Test methods}

To conduct quantitative analysis assessing the accuracy of the DEM simulation, using the same parameters, undersized material was collected along the length of the vibrating screen and its distribution could be examined. Comparing values obtained from the DEM simulation analysis, the accuracy of the simulation was verified. The following parameters were set: amplitude, $25 \mathrm{~mm}$; screen surface inclination, $4^{\circ}$; and frequency, $4 \mathrm{~Hz}$. The software EDEM 2.3 was used to simulate and calculate the weight percentage of different areas. After each test was finished, the undersized material in the five sampling boxes (from front to back, No. 1-5) was respectively weighed and then the weight percentage of each sample box could be calculated. The test was repeated seven times and the average of percentages could be deduced.

\subsection{Results analysis}

Experimental data and values obtained the DEM simulations are shown in Figure 5. As shown in Table 4, the mean difference was smaller for the two groups.
According to the $t$-test critical value table (Critical confidence level), $t(4,0.05)=2.78$, calculated value $t=$ $0.0073<2.78$, showed that the difference between the data of the two groups was smaller at a 0.05 significance level and the correlation coefficient of the paired sample was 0.9867. The data from the two groups were highly correlated. Thus, the DEM model and simulation parameters were confirmed as valid for further simulations.

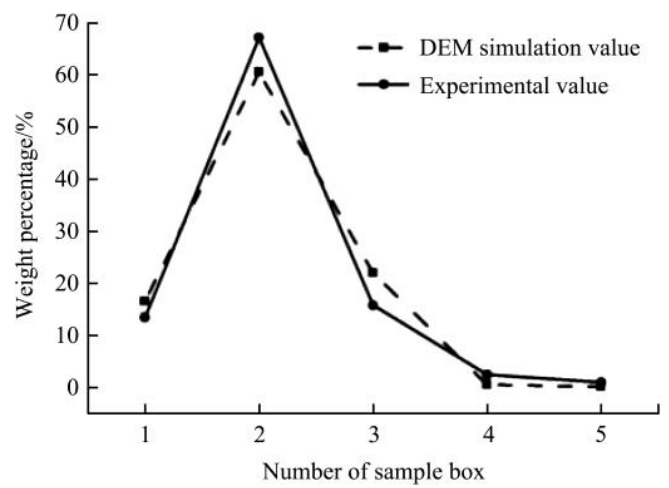

Figure 5 DEM simulation and experimental values

Table 4 Data variance analysis of the two groups

\begin{tabular}{cccccc}
\hline $\begin{array}{c}\text { Mean } \\
\text { difference }\end{array}$ & $\begin{array}{c}\text { Standard } \\
\text { deviation }\end{array}$ & Standard error & $p$ & $t$ & $d f$ \\
\hline 0.0161 & 4.9132 & 2.1972 & 0.0073 & 4 & 0.9945 \\
\hline
\end{tabular}

Note: Correlation coefficient of paired sample: $0.9867,95 \%$ confidence interval: 6.1166-6.0864.

\section{Results and analysis}

\subsection{Simulations and analysis}

Figure 6 presents the simulation results of the threshed mixture screened on the planar sieve over $0.6 \mathrm{~s}$ using the following parameters: amplitude, $25 \mathrm{~mm}$; screen surface inclination, $4^{\circ}$; and frequency, $4 \mathrm{~Hz}$. In order to allow for clear observation, the grains, shriveled grains and short stalks were respectively designated as green, yellow and blue.

Figure 6 indicates that the grain mixture entered the planar vibratory screening device at the initial velocity. Then, due to gravity, the mixture fell onto the screen surface. When the vibrating sieve motion reached $1 / 2$ cycle, the mixture gradually accumulated on the screen surface. Under the sieve's vibration force, the mixture shook and segregated as well as slid to one side due to the pushing effect of the screen surface. When the vibrating screener reached $3 / 4$ cycle, the majority of particles were 
making contact with the screen surface, and the smaller particles could be sieved through so as to produce parabolic movement. Because their size made it difficult for short stalks to pass through the sieve, they remained on the screen surface. In fact, the only means

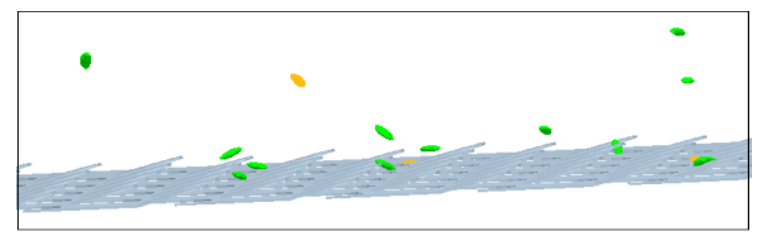

a. $0.02 \mathrm{~s}$

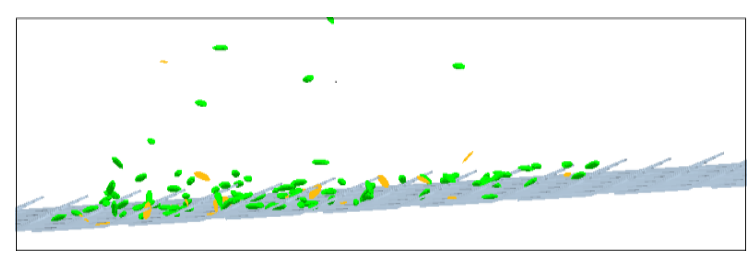

c. $0.12 \mathrm{~s}$

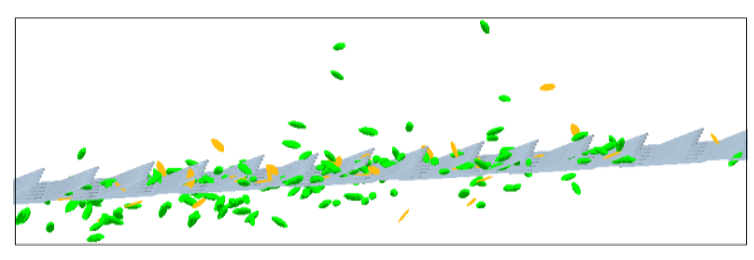

e. $0.24 \mathrm{~s}$

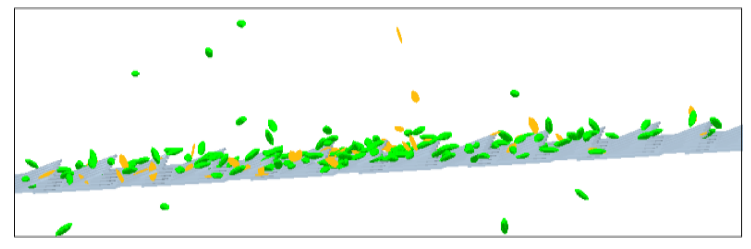

g. $0.36 \mathrm{~s}$

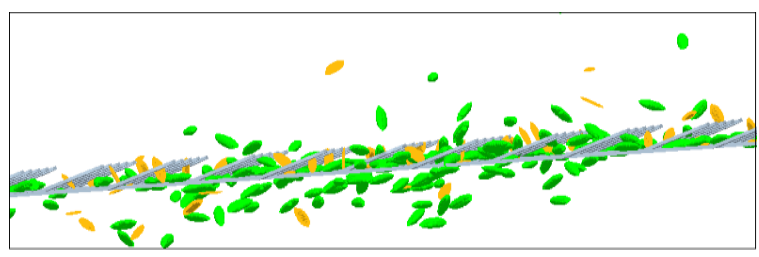

i. $0.48 \mathrm{~s}$ of separating them from the other particles was by pushing them to the rear of the sieve under the sieve's pushing effect. During the screening process, no significant difference could be observed between the movement of the grains and that of the shriveled grains.

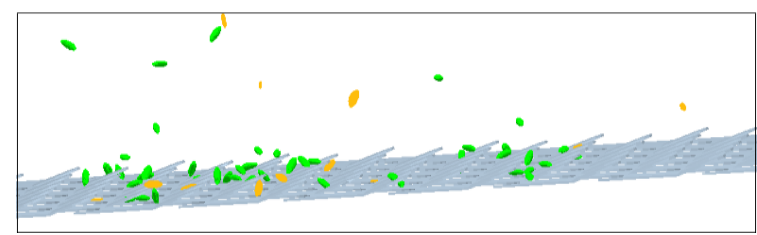

b. $0.06 \mathrm{~s}$

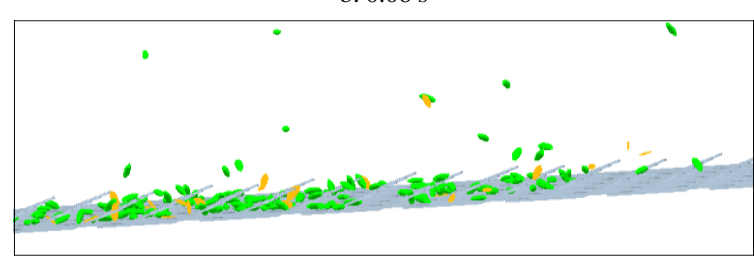

d. $0.18 \mathrm{~s}$

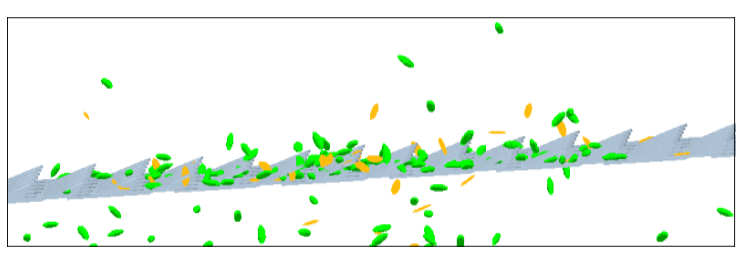

f. $0.30 \mathrm{~s}$

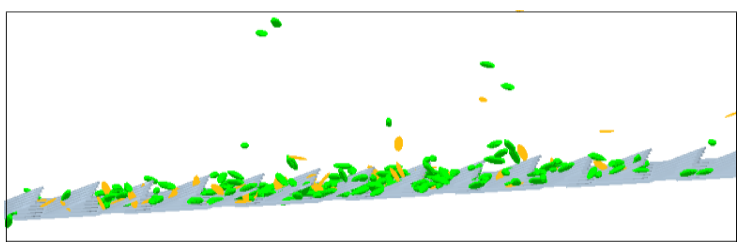

h. $0.42 \mathrm{~s}$

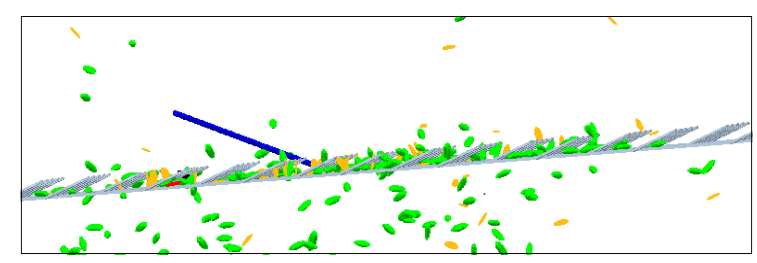

j. $0.54 \mathrm{~s}$

Figure 6 Simulation of vibrating screening

\subsection{Particle mixture motion analysis}

Figure 7 shows the detail of changes in the average impact and velocity of the grains and shriveled grains at an instance during the screening process using the following parameters: amplitude, $25 \mathrm{~mm}$; inclination, $4^{\circ}$; and frequency, $4 \mathrm{~Hz}$.

Figures 7 and 8 indicate that, after the grains and shriveled grains fell onto the screen surface, their average impact and speed periodically varied, but the cycle was consistent. Compared to the shriveled grains, the grains demonstrated a far greater average impact, and their average speed was slightly less during the screening process. The mechanism could be interpreted as follows: due to their physical properties, the grains made greater impact during the screening motion, but because of their relatively large mass, acceleration was relatively small, and their average velocity during motion was slightly less than that of the shriveled grains. Furthermore, as the average instantaneous speeds of the grains and shriveled grains were similar during motion, it was difficult to effectively separate the grains from the shriveled grains by only using the separation functions of the vibratory sieve. 


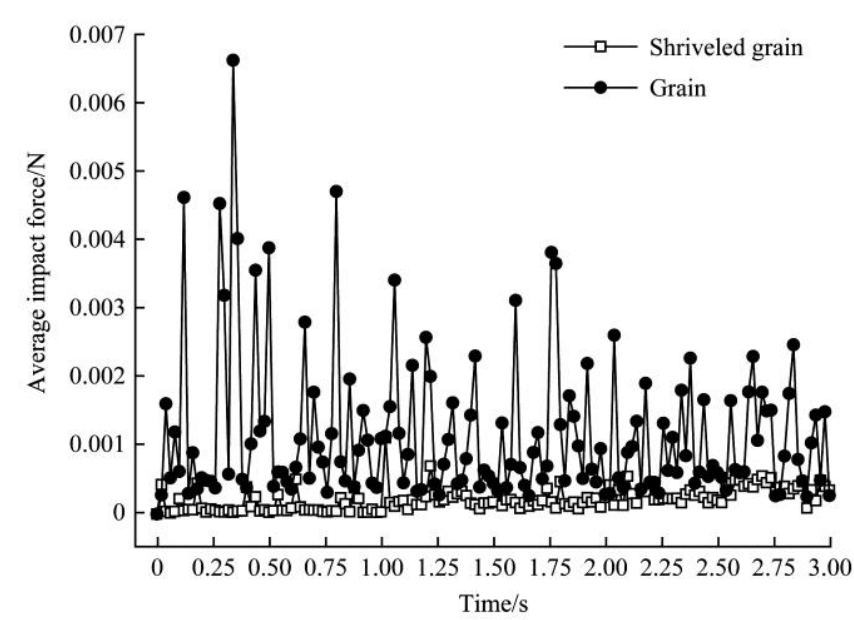

Figure 7 Change of average impact on particles

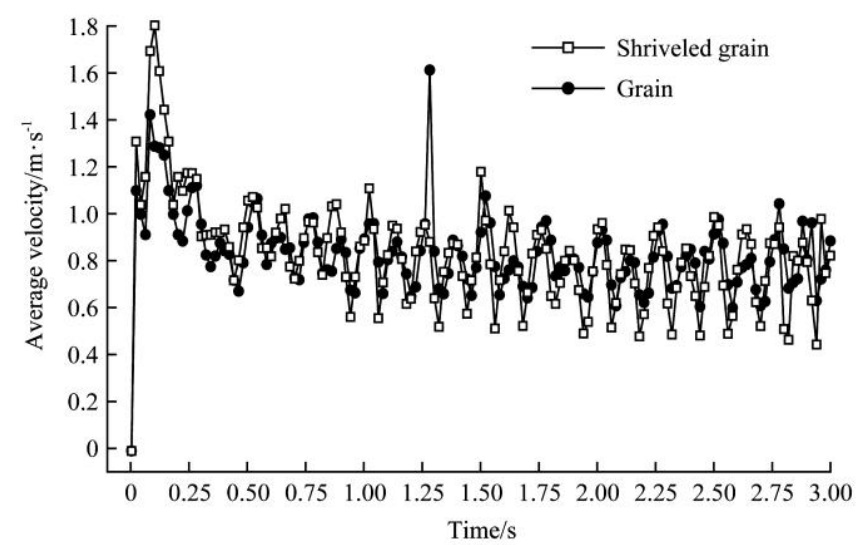

Figure 8 Change of average speed of particles

\subsection{Effect of vibration parameters on average impact of particle mixture}

For a more intuitive analysis of the effects of various factors on the average impact of the particle mixture, the results obtained from numerical calculations were further analyzed in order to identify any patterns, as shown in Figures 9-11.

Under the same conditions, the grains made a greater impact than did the shriveled grains. When the amplitude was increased, the impact exhibited a declining tendency because the greater distance the particles were pushed along the screen surface during a cycle, the smaller the change in the interaction force between the particles, and the smaller the total force. Furthermore, when the frequency was changed from $3 \mathrm{~Hz}$ to $5 \mathrm{~Hz}$, and the screen surface inclination was changed from $3^{\circ}$ to $5^{\circ}$, the change in the average impact grains made was small, thus indicating that a smaller vibration frequency and screen surface inclination slightly affected the external force that the grains were subjected. However, when the frequency was increased to $6 \mathrm{~Hz}$, the average impact on the grains was reduced; thus, this indicated that with a high frequency, the impact on the grains was offset when the grains moved back and forth on the screen surface. Furthermore, when the screen surface inclination was increased by $6^{\circ}$, the average impact on the grains significantly increased, thereby indicating that the total impact on the grain mixture increased by a large margin due to the influence of gravity at a greater tilting angle. As for the shriveled grains, when the frequency was highest $(6 \mathrm{~Hz})$ and smallest $(3 \mathrm{~Hz})$, the impact on them was greater than it was when the frequency was $4 \mathrm{~Hz}$ and $5 \mathrm{~Hz}$. The underlying mechanism could be that the mass of the shriveled grains was smaller, so when the vibration frequency was both relatively high and low, a more obvious increase in the interaction forces between the particles could be observed. The screen surface inclination had less impact on the shriveled grains, and the mechanism here was related to their smaller mass as well as the smaller effect that gravity had on the increased impact they were subjected.

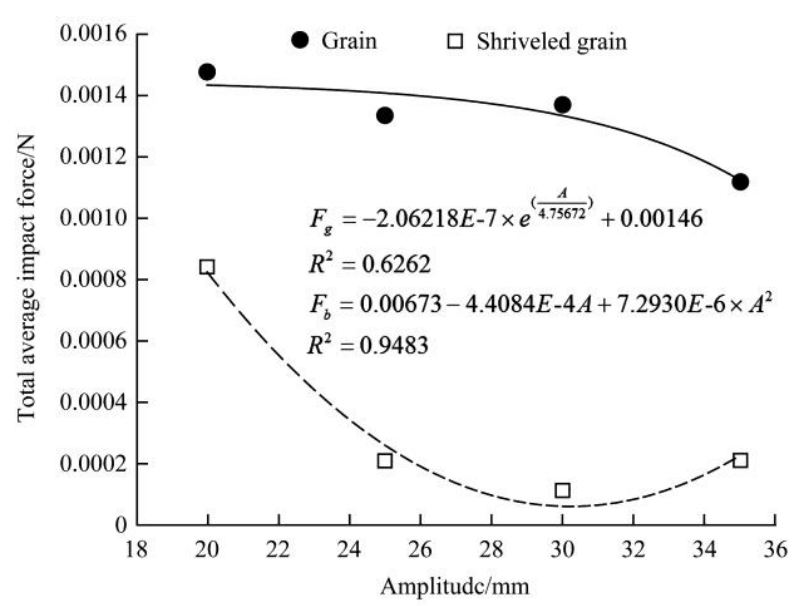

Figure 9 Average impacts on particles at different amplitudes

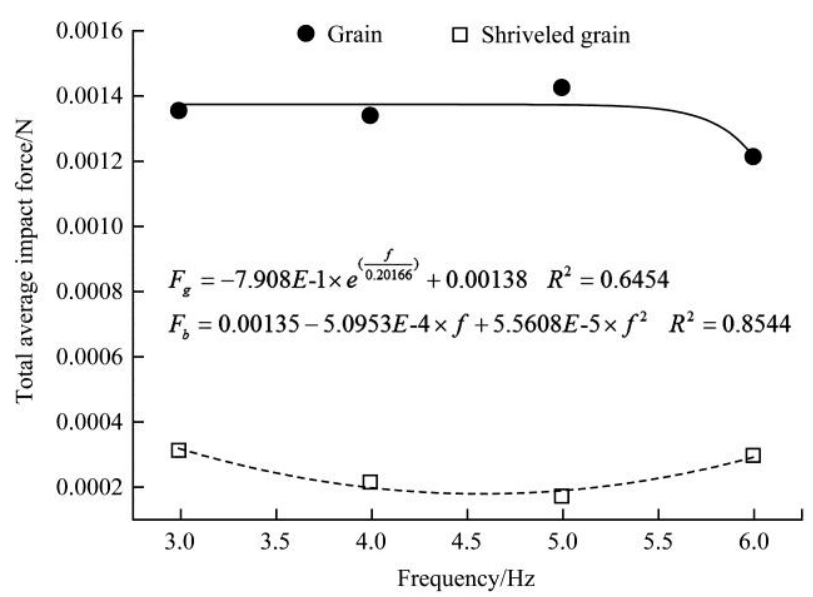

Figure 10 Average impacts on particles at different frequencies 


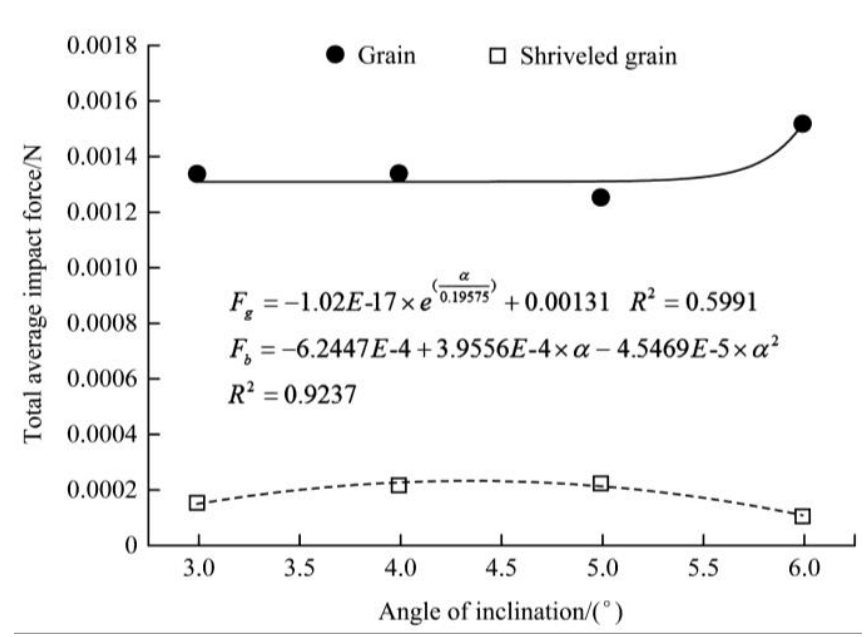

Figure 11 Average impacts on particles at different sieve angles

The relationship between the $R^{2}$ (Goodness of fit) value based on the curve fitting and the impact on shriveled grain $\left(F_{b}\right)$ experienced by the shriveled grains as well as various factors was significantly strong, whereas the variation pattern of the impact on grains $\left(F_{g}\right)$ according to various factors was not as significant after fitting.

\subsection{Impacts of various factors on average velocity of particle mixture}

After the numerical results were processed, the variation pattern of the average velocity of the grains and shriveled grains under varying factors was obtained and is shown in Figures 12-14.

Figures 12-14 indicate that, under the same conditions, the average velocity of the shriveled grains was higher than that of the grains. At amplitudes of $20 \mathrm{~mm}$ and $30 \mathrm{~mm}$, the average velocity of the particles was relatively high. Furthermore, as the frequency and the screen surface inclination increased, the average velocity of the grains and shriveled grains also showed a tendency to increase. This is because the shriveled grains had a smaller mass and could accelerate faster in the course of contact with the screen surface, and thus were faster than the grains. At a greater frequency, the acceleration of the back and forth motion of the screen surface increased, the speed of the particles increased under the pushing motion, and the overall speed increased. Be in a greater screen inclination condition, the velocity showed an increasing trend under the influence of gravity. According to the $R^{2}$ value of the curve fitting, the curve equation reflecting the relationship between the shriveled grains' velocity $\left(V_{b}\right)$ and the vibration parameters was significantly stronger, and the curve equation demonstrating the relationship between the velocity $\left(V_{g}\right)$ and the frequency of the grains and the screen surface inclination was also quite significant, whereas the curve equation between the grain velocity $\left(V_{g}\right)$ and the amplitude variation was of average significance.

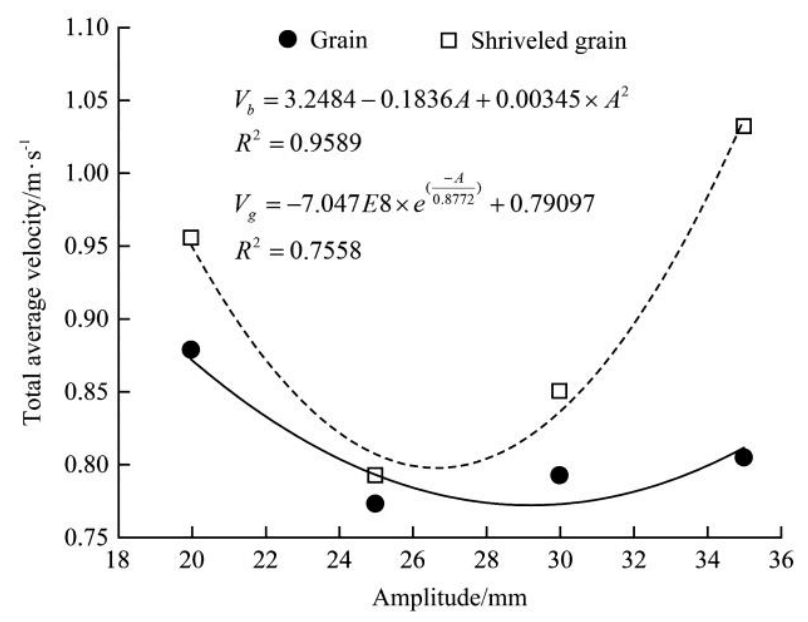

Figure 12 Average velocity of particles at different amplitudes

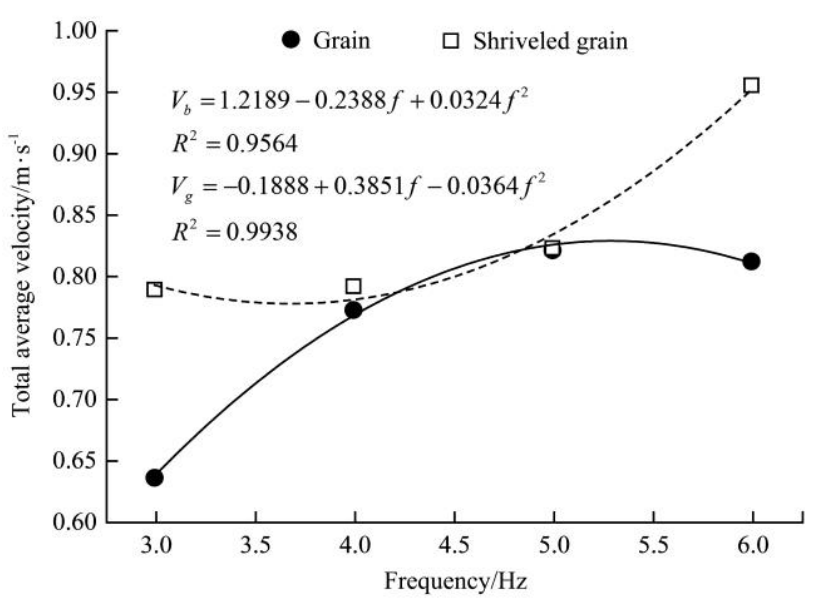

Figure 13 Average velocity of particles at different frequencies

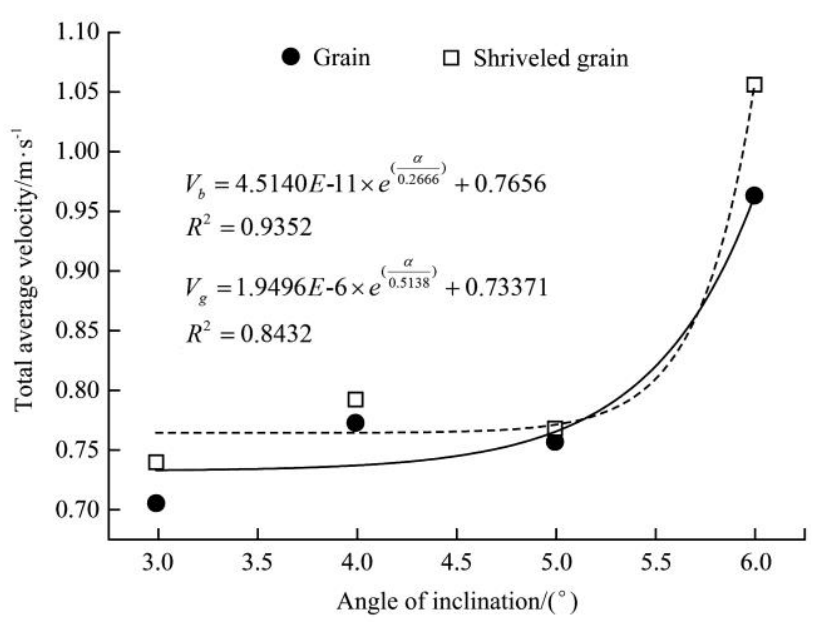

Figure 14 Average velocity of particles at different angles 


\section{Conclusions}

A 3D soft-sphere discrete element model was adopted in this work to construct a 3D discrete element model for each component of a threshed rice mixture. The screening process was numerically calculated, and the following conclusions could be made:

(1) The rice grain mixture 3D discrete element model, which was developed in this study by using bonding method, reproduced the screening process of multi-component mixtures; a multi-component mixture underwent a back and forth motion within a single cycle, and the motions of the grains and shriveled grains were found to be similar, but the short stalks were difficult to sift.

(2) When being screened with the vibratory screen, the impact that both the grains and shriveled grains was subjected to and their velocity changed periodically. The impact on the grains was much greater than it was on the shriveled grains but, during the screening process, the velocity of the grains' motion was less than that of the shriveled grains, with only a small difference existing between them.

(3) The relationship between the average impact on the particle flow and the average velocity and the vibration parameters was analyzed, and curve fitting was performed. The results revealed that the relationship between the impact on the shriveled grains and their average velocity and vibration parameters was significantly strong. It is beneficial for the separation of grains from shriveled grains when the frequency was set as $6 \mathrm{~Hz}$ and the screen surface inclination was set as $6^{\circ}$.

\section{Acknowledgement}

This work was supported by the National Natural Science Foundation of China (51305182) and the Ministry of Agriculture Key Laboratory of Modern Agricultural Equipment Grant (201303 003).

\section{[References]}

[1] Cleary P W, Sawley M L. DEM modelling of industrial granular flows: 3D case studies and the effect of particle shape on hopper discharge. Applied Mathematical
Modelling, 2002; 26(2): 89-111.

[2] Cleary P W. DEM simulation of industrial particle flows: Case studies of dragline excavators, mixing in tumblers and centrifugal mills. Powder Technology, 2000; 109(1-3): 83-104.

[3] Li J, Webb C, Pandiella S S, Campbell G M. Discrete particle motion on sieves - a numerical study using the DEM simulation. Powder Technology, 2003; 133(1-3): 190-202.

[4] Li J, Wee C, Pandiella S S, Campbell G M. A numerical simulation of separation of crop seeds by screening-effect of particle bed depth. Food and Bioproducts Processing, 2002; 2: $109-117$.

[5] Chen Y H, Tong X. Application of the DEM to screening process: a 3D simulation. Mining Science and Technology, 2009; 19: 493-497.

[6] Jiao H G, Li J R, Zhao J F, Zhao Y M. Discussion on the calculation parameters of discrete element method. Journal of Henan Polytechnic University (Natural Science), 2007; 1: 88-93. (in Chinese)

[7] Wiącek J. Geometrical parameters of binary granular mixtures with size ratio and volume fraction: experiments and DEM simulations. Granular Matter, 2016; 18(3): 42.

[8] Zhao Y M, Zhang S G, Jiao H G, Tie Z J. Simulation of discrete element of particles motion on the vibration plane. China University of Mining \& Technology, 2006; 35(5): 586-590. (in Chinese)

[9] Jiao H G, Zhao Y M. Screen simulation using a particle discrete element method. China University of Mining \& Technology, 2007; 36(2): 232-236. (in Chinese)

[10] Jiao H G, Zhao Y M. Computer emulation of particle motion on the screen plate and its validation by test. Mining \& Metallurgy, 2006; 15(1): 63-67. (in Chinese)

[11] Lenaerts B, Aertsen T, Tijskens E, de Ketelaere B, Ramon H, de Baerdemaeker $\mathrm{J}$, et al. Simulation of grain-straw separation by Discrete Element Modeling with bendable straw particles. Computers and Electronics in Agriculture, 2014; 101: 24-33.

[12] Zhao L L, Liu C S, Yan J X, Jiang X W, Zhang Y. Numerical simulation of particle segregation behavior in different vibration modes. Acta. Phys. Sin., 2010; 59(04): 2582-2588. (in Chinese)

[13] Zhao L L, Liu C S, Yan J X, Jiang X W, Zhu Y. Numerical simulation on segregation process of particles using $3 \mathrm{D}$ discrete element method. Acta Phys Sin, 2010; 59(3): 1870-1877. (in Chinese)

[14] Zhao L L, Liu C S, Yan J X, Jiang X W, Zhu Y. Numerical simulation of particles flow on the vibrating screen plate using a 3D discrete element method. China University of Mining \& Technology, 2010; 39(3): 414-419. (in Chinese) 
[15] Zhao L L, Liu C S, Yan J X, Xu Z P. Numerical simulation of particles screening process based on 3D discrete element method. China Coal Society, 2010; 35(2): 307-311. (in Chinese)

[16] Oda M, Iwashita K, Kakiuchi T. Importance of particle rotation in the mechanics of granular materials. Powders \& grains, 1997; 97: 207-210.

[17] Zhao Y Z, Jiang M Q, Xu P, Zheng J Y. Discrete element simulation of the microscopic mechanical structure in sandpile. Acta Phys Sin, 2009; 58(03): 1819-1825. (in Chinese)

[18] Li H, Yin W Q, Gao X, Liu Y. Construction of three-dimensional model of grain based on reverse engineering. Nat. Sci. Ed., 2011; 39(12): 201-206. (in Chinese)

[19] Zhou Z E. Agricultural materials. Beijing: Agriculture Press, 1994. (in Chinese) 